\title{
Mütter zu gesunder Ernährung motivieren - Langzeitfolgen fürs Kind vorbeugen
}

\author{
Franziska Scheu, Ernährungswissenschaftlerin und angehende Hebamme, Karlsruhe
}

\section{Ein nicht eingestellter Diabetes mellitus in der Schwangerschaft erhöht das Risiko für das Kind, später an Diabetes Typ 2 zu erkranken. Hebammen spielen bei der Prävention eine Schlüsselrolle. Deshalb ist es wichtig, die Mechanismen der fetalen Programmie- rung bei Gestationsdiabetes zu verstehen. Eine frühe Ernährungstherapie der Mutter kann diabetogenen Stoffwechselstörungen beim Kind vorbeugen.}

Dieser Beitrag basiert auf einer wissenschaftlichen Arbeit, die für den Bübchen Wissenschaftspreis für Hebammen 2015 eingereicht wurde.

Eine Störung des Glukosestoffwechsels in der Schwangerschaft geht mit einem Gestationsdiabetes mellitus (GDM) einher. Dass dieser erhöhte Risiken für Frühgeburtsbestrebung, intrauterinen Fruchttod, Schulterdystokie oder postnatale Hypoglykämie mit sich bringt, ist bekannt.

Doch die Folgen sind noch viel weitreichender und können das Kind bis ins Erwachsenenalter beeinträchtigen. Während der Schwangerschaft kann das intrauterine diabetische Milieu so ungünstig sein, dass es den Stoffwechsel des kindlichen Organismus nachhaltig beeinflusst und das Risiko für die Entwicklung metabolischer Krankheiten erhöht. Denn bereits beim Fetus werden grundlegende Mechanismen prägend verändert, die dann sozusagen fürs Leben „programmiert“ sind.

\section{Entstehung von Gestations- diabetes mellitus}

Während der Schwangerschaft kommt es zu enormen Veränderungen des Hormonhaushaltes, wobei vor allem die Plazenta als hochpotentes endokrines Organ in den mütterlichen Stoffwechsel eingreift. Durch die kontinuierliche $\mathrm{Zu}$ nahme an Wachstumshormonen wie Prolaktin, humanes Plazentalaktogen,
Cortisol und Progesteron sowie das Schwangerschaftshormon Östrogen nimmt die Insulinsensitivität um 50$60 \%$ ab [2]. Das sichert dem Ungeborenen die Nahrungszufuhr.

Durch genau diese natürliche Insulinresistenz wirkt Insulin vermindert am mütterlichen Zielgewebe und es bleibt mehr Glukose im Blut [25]. Dadurch kann Glukose als wichtiger Nährstoff direkt über die Plazenta zum Fetus gelangen.

Jedoch passt dieser sinnvolle natürliche Prozess nicht zu den heutigen Lebensstilfaktoren: Ein Nahrungsüberangebot und eine unkritische Ernährungsweise (hohe Energie- und Fettaufnahme) spielen eine grundlegende Rolle bei der chronischen Insulinresistenz und führen bereits vor der Schwangerschaft zur ßZell-Dysfunktion.

In Kombination mit der natürlichen und charakteristischen Insulinresistenz der Schwangerschaft kommt es zu einem additiven Effekt. Das führt häufig zu einem Gestationsdiabetes mellitus.

Bei GDM ist die Insulinresistenz übermäßig ausgeprägt und es kommt zu einer pathologischen Glukosetoleranzstörung, wobei die Hyperglykämie in ihrer Intensität variabel ist [34]. Im ersten Schwangerschaftsdrittel sollte eine Risiko- evaluierung stattfinden (siehe Kasten „Screening und Diagnostik“).

\section{Was heißt fetale Programmierung?}

Mittlerweile gibt es eine Vielzahl an Begriffen für die Prägung im Mutterleib wie Fetale Programmierung, Nutritional Programming, Metabolic Imprinting, Functional Neuroteratology oder Fuel Mediated Teratogenesis. Alle diese Begriffe umschreiben eine zeitlich begrenzte, sensible Phase, in der Faktoren wie Ernährung oder Hormone auf das heranwachsende Kind und seinen Organismus einwirken und eine physiologische Adaptation an die aktuell erfahrenen Bedingungen ermöglichen. Dieser Mechanismus kann aus evolutionsbiologischer Sicht einen Überlebensvorteil bewirken [22].

Solche Prägungs- und Anpassungsleistungen können jedoch auch fatale körperliche Folgen in späteren Lebensphasen haben [31]. Ungünstige Bedingungen im Mutterleib gehen mit einem erhöhten metabolischen Krankheitsrisiko des Kindes im späteren Alter einher.

Klinische, epidemiologische und experimentelle Daten zeigen, dass ein mütterlicher Gestationsdiabetes mit 


\section{GDM - Screening und Diagnostik}

Die International Diabetes Federation (IDF) stellte 2010 fest, dass ein GDM mit Komplikationen in der Schwangerschaft assoziiert ist, welche durch rechtzeitige Diagnostik und intensive Behandlung abgemildert werden können. Zur Diagnose wurde deshalb im Jahr 2012 der oGTT (oraler Glukosetoleranztest) (Tab. 1) in die regulären Untersuchungen des Mutter-Kind-Passes aufgenommen. Der 75g-oGTT ist der Goldstandard für die Diagnose von Gestationsdiabetes, wird jedoch in der Praxis oft als zu aufwendig betrachtet.

Deshalb werden zunächst andere Methoden wie das Screening mittels 50-g-Suchtest (Glucose Challenge Test, GCT) durchgeführt. Dieser ist unabhängig von der Tageszeit und der Nahrungsaufnahme durchführbar. Ist der Blutzuckerwert eine Stunde nach dem Trinken der Testlösung $\geq 135 \mathrm{mg} / \mathrm{dl}$ (7,5 mmol/l), gilt er als positives Screening und erfordert einen anschließenden diagnostischen 75g-oGTT [21].

Ein „Gestationsdiabetes mellitus“ ist nach dem Diagnoseschlüssel (GDM, ICD-10: O24.4G) bereits dann definiert, wenn nach einem 75-g oralen Glukosetoleranztest (oGTT) unter standardisierten Bedingungen und qualitätsgesicherter Glukosemessung aus venösem Plasma ein erhöhter Glukosewert vorliegt.

Besonders hoch ist das GDM-Risiko für werdende Mütter

- über 45 Jahren

- bei bestehenden Diabetes-Symptomen

- Prädiabetes in der Anamnese (gestörte Glukosetoleranz und/oder Nüchternglukose $>100 \mathrm{mg} / \mathrm{dl}$ )

- Geburt eines Kindes mit $>4.500 \mathrm{~g}$

- Adipositas, metabolischem Syndrom

- habituellem Abortus

Zwischen der 24.-28. SSW wird dann ein zweistündiger 75g-oGTT durchgeführt. Der Test findet also im zweiten
Trimenon statt, da in der Regel die Kapazität der mütterlichen Bauchspeicheldrüse erst zu diesem Zeitpunkt überfordert ist [18]. Im Jahr 2013 ergab sich in Deutschland nach der Perinatalstatistik eine GDM-Prävalenz von 4,4\% (29.103 Fälle) [20].

Eine Diabetes-Diagnostik vor der 24. SSW macht man aufgrund vorliegender Risiken:

- Alter $>45$ Jahre

- präkonzeptioneller BMI $>30 \mathrm{~kg} / \mathrm{m}^{2}$

- körperliche Inaktivität

- familiäre Diabetesbelastung

- Geburt eine Kindes $>4500 \mathrm{~g}$

- RR > 140/90 oder Einnahme von Antihypertensiva

- Dyslipidämie

- Polyzystisches Ovarialsyndrom

- Prädiabetes in der Anamnese

- klinische Insulinresistenz

- KHK, pAVK, zerebral-arterielle Makroangiopathie

- Kontrainsulinäre Medikation (z.B. Glukokortikoide)

Tab. 1 Grenzwerte für die Diagnose eines GDM [27].

Grenzwerte für die Diagnose von GDM (gesamte Schwangerschaft, oGTT vorzugsweise in der 24.-28. SSW)

Nüchtern $\geq 92 \mathrm{mg} / \mathrm{dl}$

$1 \mathrm{~h}$ postprandial $\geq 180 \mathrm{mg} / \mathrm{dl}$

2 h postprandial $\geq 153 \mathrm{mg} / \mathrm{dl}$

Diabetes Diagnose (vor der 20. SSW)

Nicht nüchtern $\geq 200 \mathrm{mg} / \mathrm{dl}$

Nüchtern einer Insulinerhöhung beim Feten einhergeht und offenbar als prädisponierender, fehlprogrammierender Faktor lebenslang eine erhöhte Adipositasund Diabetesdisposition zur Folge hat.

Inzwischen belegen nicht nur Tierstudien, sondern auch umfangreiche epidemiologische und humane Interventionsstudien, dass ein evidenter Zusammenhang zwischen frühester Ernährung und späterer Adipositas sowie damit assoziierten Erkrankungen im Erwachsenenalter besteht $[16,23]$.

\section{Weitere Risikofaktoren für Diabetes}

Es gibt verschiedene Einflussfaktoren, die das Risiko für Diabetes erhöhen. Eine große Rolle spielen Umwelteinflüsse, Lebensstil, Übergewicht und Adipositas, Gene und damit assoziiert die nichtübertragbaren Erkrankungen, akzelerierte postnatale Gewichtszunahme, Fehlanpassung des prä- und postnatalen Wachstums sowie besonders die fetale überversorgung [22]. Auf Letzteres wird folgend besonders Augenmerk gelegt, denn für eine gesunde Entwicklung des
Föten, Kindes und Jugendlichen hat eine optimale Versorgung während der Schwangerschaft hohen Stellenwert [18].

\section{Was passiert in der kritischen Phase?}

Durch das neuroendokrine Immunsystem werden fundamentale Lebensprozesse wie Fortpflanzung, Wachstum und Informationswechsel geregelt. Darunter versteht man die Gesamtheit von vielen Subsystemen, deren Zusammenspiel von chemischen Botenstoffen regu- 
liert wird, die zudem mit der Umwelt interagieren.

Hormone übertragen Informationen von der Umwelt an den Organismus, besonders an das Genom und Gehirn - quasi als endogene Effektoren. Alle Organbestandteile (Zellen, Organe) sind gemeinsam an der Prozessierung des Hormonsystems beteiligt.

Wichtig ist die Sekretion von Hungerund Sättigungshormonen wie Adiponektin, Leptin, Neuropeptid. Es ist also besonders bedeutsam, wie intrauterine Hormone und alle chemischen Signalstoffe auf den sich entwickelnden, sich selbst organisierenden Organismus wirken $[28,14]$.

Das vegetative Nervensystem regelt die Stoffwechselorgane bzw. -systeme und unterliegt dem sogenannten Regelkreis - mit dem Hypothalamus als übergeordnetem Regler. Die Regelgröße wird durch diesen zentralnervösen Regler mithilfe eines Erfolgssignals beeinflusst. Er ist aber in der kritischen Entwicklungsphase noch nicht ausgereift. Die mütterlichen Stoffwechselprodukte und der aktuelle Spiegel der Hormone werden über die Plazenta zum Kind gebracht und beeinflussen seine fetalen Stoffwechselorgane.
Diese Hormone und Metabolite wirken auf das Gehirn programmierend und agieren im späteren Leben als sekundäre Regelgrößen. Denn Nervenzellen, die sich fetal entwickeln, erfahren den aktuellen Spiegel ihrer eigenen späteren Regelgrößen. So kommt es zu einer dauerhaften Fehlorganisation und zu dauerhaften Störungen der Regelsysteme $[7,6]$.

\section{Kompensationsstrategien des Fetus}

Der Fetus muss die hohen Zuckerspiegel der Mutter durch eigene erhöhte Insulinausschüttung kompensieren. Auf diese Weise werden bei dauerhaftem Überangebot an Glukose, die ß-Zellen der Bauchspeicheldrüse stimuliert und schütten die entsprechende Menge an Insulin aus. Im Gehirn wird dieser „Sollwert an Insulin“ als primäres Steuerungssystem für die eigene spätere Regulation determiniert. Sekundär, das heißt jenseits der kritischen Entwicklungsphase, ist dadurch die zu regelnde Insulingröße bereits festgeschrieben [8]. Ist der programmierte Sollwert zu hoch, resultieren häufig diabetische Stoffwechselstörungen [25]. Eine solch erworbene Fehlprogrammierung hat poten- zielle Langzeitfolgen mit dem Risiko, selbst an Diabetes zu erkranken.

\section{Welche Rolle spielt das Gehirn?}

Der Hypothalamus scheint ziemlich sensibel für eine Programmierung zu sein $[32,13]$. Deshalb können Veränderungen der Hormone und Rezeptoren in dieser Gehirnregion enormen Einfluss auf das Appetitverhalten ausüben. Übergewicht und Fettleibigkeit durch „Overeating“ sind die Folge.

In einem Tierexperiment mit Ratten wurde untersucht, ob tatsächlich das Gehirn die zentrale Rolle bei diesem Geschehen spielt. Dazu wurden während der Differenzialphase in der Neugeborenenentwicklung höhere Insulinkonzentrationen in den neonatalen Hypothalamus appliziert. Dies sollte eine hohe Insulinsekretion des Feten bei einem GDM nachahmen. Die Tiere und ihre sich entwickelnden Nervenzellen wurden diesem Spiegel während der kritischen Entwicklungsphase für zwei bis drei Tage ausgesetzt. Im späteren Leben entwickelten die Ratten all das, was mit dem metabolischen Syndrom zusammenhängt: Übergewicht, eine gestörte

\section{Studien}

- Silvermann et al. untersuchten 1996 die Häufigkeit einer gestörten Glukosetoleranz bei Nachkommen von amerikanischen Müttern mit klinischen Symptomen eines GDM einerseits und mit einem eingestellten insulinpflichtigen Diabetes andererseits. Gemessen wurde der Fruchtwasserinsulinspiegel zwischen der 32. und 38. SSW. Die 88 Kinder von nichteingestellten GDM-Müttern (zeigten erhöhten Fruchtwasserinsulingehalt, was typisch ist für einen fetalen Hyperinsulinismus) wurden mit den 80 Kindern aus der Kontrollgruppe (normaler Fruchtwasserinsulingehalt) verglichen. 19,3\% der Kinder, die intrauterinem Diabetes ausgesetzt waren, entwickelten im Alter von 10 bis 16 Jahren eine gestörte Glukosetoleranz. Bemerkenswert war, dass Kinder mit erhöhtem Fruchtwasserinsulinspiegel auch im viel späteren Alter (>16 Jahre) höhere Glukose- und Insulinwerte hatten. 33,3\% von ihnen zeigten gestörte Glukosetoleranzwerte - bei denen mit normalem Fruchtwasserinsulingehalt lag das Risiko nur bei 3,7\%. Diese Befunde verdeutlichen einen starken Zusammenhang zwischen diabetischem Milieu während der kritischen Entwicklungsphase und dem Risiko einer Entwicklung von diabetogenen Stoffwechselstörungen [33].

- Auch eine Studie von Dabela et al. von 2000 mit PimaIndianern, einer besonders exemplarischen Risikopopulation für die Entwicklung eines Typ-2-Diabetes, ist erwähnenswert: Evaluiert wurden Geschwister, von denen eines vor der mütterlichen Diagnose eines Typ-2-Diabetes geboren wurde und eines nach der Diagnose. Neben einem deutlich erhöhten BMI im Erwachsenenalter lag nur bei jenen Kindern, die intrauterin einem mütterlichen Diabetes ausgesetzt waren, das Risiko zur Entwicklung eines Typ-2-Diabetes sehr viel höher und ging sowohl mit Störungen im Glukosestoffwechsel als auch mit Störungen der Insulinsekretion und -sensitivität einher [5]. 


\section{Tipps aus der Ernährungstherapie}

Wenn bei einer Frau Gestationsdiabetes festgestellt wird, folgt in der Regel die Überweisung zu einer DiabetesSchwerpunkteinrichtung, wo sie an einer Schulung für Schwangere teilnimmt.

Im Gespräch wird herausgefunden, was die Frau gewöhnlich isst und trinkt, was ihr schmeckt, wie der Tagesablauf mit den Mahlzeiten aussieht, ob sie eine besondere Ernährungsform bevorzugt (z.B. vegetarisch), welche Vorlieben sie hat, sowie ob kulturelle und religiöse Aspekte oder persönliche Einstellungen zum Essen zu berücksichtigen sind. Die Ernährungsberatung wird individuell mit ihrer Alltagssituation verknüpft, zum Beispiel mit einer Berufstätigkeit. In der Beratung wird gezeigt, welche Nahrungsmittel den Blutzucker verändern und wie sie zuckerhaltige Speisen und Getränke gleichmäßig über den Tag verteilt werden können [21].

Die Einteilung der Makronährstoffe entspricht grob den normalen Empfehlungen für die Gesamtbevölkerung. Idealerweise liegen die prozentualen Energieanteile bei:

- Kohlenhydrate: 40-50\%

- Protein: $20 \%$

- Fett: $30-35 \%$

Diabetikern wird also keine besondere Diät oder andere Ernährungsform empfohlen. Die Schwangere kann sich wie ihre Familie oder ihr Freundeskreis ernähren, sollte dabei jedoch ein paar Grundregeln für eine gesunde Ernährung befolgen. Ziel ist es, die Entstehung einer dauerhaften Zuckerkrankheit zu verhindern.

\section{Mehrere kleine Mahlzeiten am Tag}

Statt drei große Hauptmahlzeiten besser sechs kleinere Mahlzeiten über den Tag verteilt essen. Das verhindert einen hochschnellenden Blutzuckerspiegel nach einer großen, üppigen Mahlzeit. Neben Frühstück, Mittagessen und Abendessen also zwei bis drei Zwischenmahlzeiten einnehmen, zum Beispiel:

- 1 Naturjoghurt/Quark mit frischem Obst

- 1 Vollkornmüsli (ungezuckert) mit Milch

- 1 Scheibe Vollkornbrot mit Streichkäse und Radieschen

- 1 Portion Frischobst

\section{TIPPS}

- Bei der Arbeit kleingeschnittenes Gemüse (Radieschen/ Gurke/Karotten) griffbereit halten, dazu einen leckeren Joghurt-Kräuterdip.

- Eine kohlenhydratreiche Spätmahlzeit essen, um einer Unterzuckerung in der Nacht vorzubeugen [1].

\section{Ballaststoffreiche Lebensmittel}

Kohlenhydratreiche Lebensmittel bevorzugen (40\% der Nahrungsenergie aus Kohlenhydratenergie) - am besten sind die ballaststoffreichen Varianten: Vollkornprodukte wie z.B. Vollkornbrot, Vollkornnudeln, Getreideflocken im Müsli, grobkernige Haferflocken, unpolierter Vollkornreis, Hülsenfrüchte, Gemüse und Obst. Aufgrund ihres hohen Ballaststoffgehaltes werden sie langsamer verdaut, sodass die enthaltenen komplexen Kohlenhydrate verzögert ins Blut gelangen. Weißbrot und alle anderen ballaststoffarmen Getreideprodukte veranlassen einen schnellen Blutzuckeranstieg und sollten deshalb vermieden werden. Mit diesem Wissen lassen sich durch die Auswahl ballaststoffreicher Nahrungsmittel die Blutzuckerwerte nach der Mahlzeit gut verbessern.

\section{TIPPS}

- Kohlenhydratlastige Nahrungsmittel mit Eiweißträgern wie fettarmer Milch und fettarmen Milchprodukten, Fleisch, Fisch und Eiern kombinieren.

- Die Frau befragen, welches Brot oder Müsli sie gewöhnlich isst. Tab. 2 zeigt weitere abwechslungsreiche Vorschläge.

Tab. 2 Tipps für ballaststoffreiche Lebensmittelvariationen [12].

\begin{tabular}{|l|l|}
\hline Lebensmittel & Vollkornvariante \\
\hline Frühstückszerealien & $\begin{array}{l}\text { Haferbrei (Porridge) aus Rollhafer } \\
\text { oder Hafergrütze, Vollkorngetreide, } \\
\text { Gepufftes Vollkorngetreide, Voll- } \\
\text { kornmüsli und Getreideriegel }\end{array}$ \\
\hline
\end{tabular}

Brot und Kräcker Roggenbrot (Pumpernickel), Vollkorn-, Weizenkeim- oder Mehrkornbrote, Vollweizenkräcker, Roggenkräcker, Knäckebrot, Vollkornreiswaffeln, Haferkekse

Mehl

Vollkornmehl, Weizenkeime, Buchweizenmehl, nicht raffiniertes Roggen- oder Gerstenmehl, Haferflocken, Hafermehl

Beilagen

Brauner Reis, Vollweizenpasta, Vollkorngerste, Bulgur (gebrochener Weizen), Quinoa, Perlgerste

Lebensmittel
Vollkornvariante 


\section{Weniger Zucker}

Normaler Haushaltszucker enthält sogenannte kurzkettige Kohlenhydrate. Der deutsche Durchschnittsbürger konsumiert täglich ca. $100 \mathrm{~g}$ davon, deutlich mehr als von der WHO empfohlen. Deshalb besser nur $25 \mathrm{~g}$ täglich essen, das entspricht etwa 6 Teelöffeln Kristallzucker oder 8 Stück Würfelzucker. Achtung - Zucker steckt auch in anderen Lebensmitteln:

- Zucker in Getränken: Um raschem stark ansteigendem Blutzuckeranstieg vorzubeugen, auf Limonade, Cola ( $250 \mathrm{ml}$ beinhalten $27 \mathrm{~g}$ Zucker!), fertigen Eistee und anderen mit Zucker oder Honig gesüßte Getränke verzichten. Stattdessen reichlich Wasser, ungesüßten Früchte- oder Kräutertee oder stark verdünnte Fruchtsäfte trinken.
- Zucker in Fertiggerichten: Es ist erschreckend, wie viel Zucker in Fertigprodukten steckt. Ein Esslöffel Ketchup enthält zum Beispiel einen Teelöffel Zucker. TIPP: Lieber selbst kochen: Buntes, frisches Gemüse selbst zubereiten und mit Kräutern würzen, als Beilage, Auflauf, Eintopf, Suppe oder Salat - da ist bestimmt kein Zucker drin.

- zu viel Zucker in Süßwaren: Bonbons, Fruchtgummis und Lakritz lieber meiden. Kleine Mengen Süßwaren, also z.B. mal ein kleines Stück Kuchen oder ein Riegel Schokolade, sind nicht problematisch.

\section{TIPP}

Für süße Naschkatzen: Gelegentlich als Alternative Süßungsmittel, also kalorienfreien Süßstoff verwenden. Vernünftige Mengen Süßstoff sind auch für das Kind unschädlich.
Glukosetoleranz und eine basale Hyperinsulinämie [30, 17].

Damit erklärt sich der zugrundeliegende pathologische Mechanismus: Normalerweise reguliert der ventromediale Nukleus des Hypothalamus (VMN) das Gefühl der Sättigung und der Laterale Hypothalamus Area (LHA) steuert das Hungergefühl und den Appetit.

Die Exposition der hohen Insulinspiegel führt dazu, dass die Zellzahl in dieser Gehirnregion vermindert ist und lebenslang unterentwickelt bleibt. Die Nervenzellen sind verkleinert, hypoplastisch und hypotroph. Es kommt zu einem Ungleichgewicht und dadurch zu einer Störung des Hunger- und Sättigungsgefühls.

Es handelt sich also um eine perinatale Programmierung als Teilmechanismus einer metabolischen Prägung [14].

\section{Übergewicht und Fett- leibigkeit der Nachkommen}

Um eine gesunde Entwicklung des Fötus, Kindes und schließlich auch Jugendlichen zu gewährleisten, hat eine optimale Versorgung während der Schwan- gerschaft in Bezug auf metabolische Faktoren einen hohen Stellenwert [18]. Das derzeit größte Forschungskonsortium zur frühen Programmierung untersucht weltweit, wann genau der Körper für eine solche Programmierung sensibel ist und welche Effekte die frühe Ernährung und der Lifestyle auf die metabolische Programmierung sowie daraus resultierendes Übergewicht haben. In dem von der EU geförderten EarlyNutritionProjekt [9] arbeiten seit Februar 2012 internationale Arbeitsgruppen aus 12 europäischen Ländern wie auch den USA und Australien zusammen.

Nachkommen von nicht eingestellten diabetischen Müttern haben ein höheres Risiko, größere BMI zu entwickeln. Bereits Neugeborene von Müttern, die eine leichte Glukosetoleranzstörung haben, zeigten unabhängig vom Geburtsgewicht einen $20 \%$ höheren Körperfettanteil auf als Kinder von Frauen mit einer normalen Glukosetoleranz [4].

Grillmann et al. fanden 2003 heraus, dass Kinder von GDM-Müttern ein 30\% erhöhtes Risiko hatten, übergewichtig zu werden, unabhängig vom sozioökonomischen Status, dem Geburtsgewicht und nachdem man die Energiebalance kontrollierte [15].
Nicht nur bei den Neugeborenen gibt es diese Tendenz zu Übergewicht, sondern auch im jugendlichen Alter gibt es den Zusammenhang zwischen GDM und Übergewicht bzw. Fettleibigkeit [26].

Problematisch ist die Fettverteilung der Kinder. Generell gilt viszerales Fettgewebe (Bauchfett) als besonders stoffwechselaktiv und ist um die Organe herum angelegt. Deshalb gilt es als ein erhöhtes Risiko für kardiovaskuläre Erkrankungen und das metabolische Syndrom mit resultierender Insulinresistenz [11].

Dass es ungünstige Fettgewebsverteilungsmuster gibt, zeigte sich in einer multiethnischen retrospektiven Kohortenstudie mit 461 Kindern zwischen 6 und 13 Jahren. Bei Kindern, deren Mütter einen GDM hatten, zeigten sich eine höhere Ansammlung an subkutanem abdominellem Fettgewebe (Magnetresonance Messung), erhöhte Körperfettanteile (Hautfaltendickenmessungen), ein größerer Taillenumfang sowie ein höherer BMI [3]. Auch in einer longitudinalen Fall-Kontroll-Studie mit 189 Mutter-Tochter-Paaren wurde gezeigt, dass GDM-Nachkommen mit 15 Jahren eine zweifach erhöhte Prävalenz hatten, übergewichtig zu sein. Außerdem lag das Risiko bei $40 \%$, ein viszerales Fettvertei- 
lungsmuster zu haben. Besonders wenn die Fettansammlung im Bereich des Abdomens auftrat („Apfeltyp“), gab es negative Zusammenhänge mit dem Nüchterninsulinlevel und niedrigeren HDL-Cholesterollevel (high-density lipoprotein) [10].

Das adipozytäre Gewebe ist ein hochaktives endokrines Organ, welches mit einer erhöhten Anzahl an zirkulierenden Metaboliten, inflammatorischen Zytokinen und Wachstumsfaktoren einhergeht [19].

\section{Hohe Bedeutung der Hebamme}

Wer Frauen mit einem GDM in der Schwangerschaft begleitet, kann diesen helfen, die Ernährung im Griff zu halten. Bei manchen wird dennoch zusätzlich eine Insulingabe erforderlich. Es ist wichtig, diese Frauen da abzuholen, wo sie stehen. Das setzt das Wissen um die Bedeutung eines unbehandelten GDM voraus, sowohl kurzfristig als auch langfristig, mit all seinen Konsequenzen vor allem für das Ungeborene und sein späteres Leben. Hebammen haben eine Schlüsselrolle: Sie können betroffene Frauen unterstützen, sich trotz GDM wohlzufühlen, und sie können sie motivieren, sich gesund zu ernähren.

Es ist wichtig, die Frauen zu ermutigen und ihnen bereits vor der ersten Schulung in einer Diabeteseinrichtung zu vermitteln, dass es um ein Training zur Selbstbehandlung geht und dass sie damit sich selbst und dem Fetus Gutes tun.

Denn eine bewusste Ernährung bietet die Chance, dass dem Kind später Insulinspritzen erspart bleiben und es mit einem nicht diabetogenen Stoffwechsel gesund gedeihen kann.

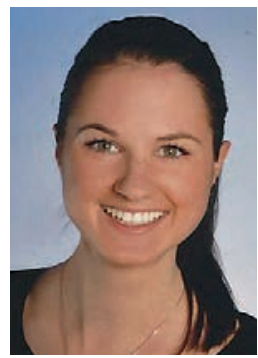

Franziska Scheu

Ernährungswissenschaftlerin und angehende Hebamme Diakonissenkrankenhaus Karlsruhe Diakonissenstraße 28 76199 Karlsruhe E-Mail: Franziska.Scheu@web.de 


\section{Literatur}

1 Bantle JP, Wylie-Rosett J, Albright AL, Apovian M, Clark NG, Franz MJ, Hoogwerf BJ, Lichtenstein AH, MayerDavis E, Mooradian AD, Wheeler ML. Nutrition recommendations and interventions for diabetes: a position statement of the American Diabetes Association. Diabetes Care 2008; 31 (Suppl 1): 61-78

2 Catalano PM, Hoegh M, Minium J, Huston-Presley L, Bernard S, Kalhan S et al. Adiponectin in human pregnancy: implications for regulation of glucose and lipid metabolism. Diabetologia. 2006; 49: 1677-1685

3 Crume TL, Ogden L, West NA, Vehik KS, Scherzinger A, Daniels S, McDuffie R, Bischoff K, Hamman RF, Norris JM, Dabelea D. Association of exposure to diabetes in utero with adiposity and fat distribution in a multiethnic population of youth: the Exploring Perinatal Outcomes among Children (EPOCH) Study. Diabetologia 2010; 54: 87-92

4 Dabelea D, Hanson RL, Lindsay RS, Pettitt DJ, Imperatore G, Gabir MM, Roumain J, Bennett PH, Knowler WC. Intrauterine exposure to diabetes conveys risks for type II diabetes and obesity: a study of discordant sibships. Diabetes 2000; 49: 2208-2211

5 Dabela D. The predisposition to obesity and diabetes in offspring of diabeticmothers. Diabetes Care 2007; 30 (Supp 2): 167-174

6 Dörner G (a) (1975) Perinatal hormone levels and brain organization. In: Stumpf WE, (Grant LD;Hrsg.), Anatomical neuroendocrinology. Karger, Basel 1975, 245-252

7 Dörner G (b), Problems and terminology of functional teratology, Acta Biol Med Ger 1975; 34 (6): 1093-1095

8 Dörner G, Plagemann A. Perinatal hyperinsulinism as possible predisposing factor for diabetes mellitus, obesity, and enhanced cardiovascularrisk in later life. Horm Metab Res 1994; 26: $213-221$

9 EarlyNutrition Project. Long-term effect of early nutrition on later health. This project receives funding from the European Union Seventh Frame-work Programme (FP7/2007-2013) under grant agreement $\mathrm{n}^{\circ} 289346$ Internet: http://www.projectearlynutrition. eu/ pdf/news/EN_factsheet_en_AL_290312. pdf (Stand:07.09.2013)
10 Egeland GM, Meltzer SJ. Following in mother's footsteps? Mother-daughter risks for insulin resistance and cardiovascular disease 15 years after gestational diabetes. 55 Diabetes Med 2010; 27: 257-265

11 Elmadfa I. Ernährungslehre, 2. Auflage, Verlag Eugen Ulmer GmbH\&Co, Stuttgart, 2009

12 EUFIC - The European Food Information Council, DIE GRUNDLAGEN 01/2009, Fakten über Vollkorn, Internet: http:// www.eufic.org/article/de/expid/ fakten-ueber-vollkorn/,2009 (Stand 22.12.2015)

13 Fisher RE, Steele M, Karrow NA. Fetal programming of the neuroendocrineimmune system and metabolic disease. J Pregnancy 2012; 2012: 792934

14 Franke K, Harder T, Aerts L, Melchior K, Fahrenkrog S, Rodekamp E, Ziska T, Van Assche FA, Dudenhausen JW, Plagemann A. „Programming“ of orexigenic and anorexigenic hypothalamic neurons in offspring of treated and untreated diabetic mother rats. Brain Res. 2005; 1031(2): 276-283

15 Gillman MW, Rifas-Shiman S, Berkey CS, Field AE, Colditz GA. Maternal gestational diabetes, birth weight, and adolescent obesity. Pediatrics 2003; 111: e221-226

16 Godfrey KM, Gluckman PD, Hanson MA. Developmental orgins of metabolic disease: life course and intergenerational perspectives. Trends Endocrinol Metab 2010; 21: 199-205

17 Hivert MF, Sullivan LM, Fox CS, Nathan DM, D'Agostino RBS, Wilson PW, Meigs JB. Associations of adiponectin, resistin, and tumor necrosis factor-alpha with insulin resistance. J Clin Endocrinol Mctab. 2008; 93: 3165-3172

18 Kautzky-Willer A, Bancher-Todesca D, Pollak A, Repa A, Lechleitner M, Weitgasser R. Gestationsdiabetes (GDM). Wien Klin Wochenschr 2012; 124 (Suppl 2): 58-65

19 King JC. Maternal obesity, metabolism, and pregnancy outcomes. Annu Rev Nutr 2006; 26: 271-291
20 Kleinwechter H, Schäfer-Graf U, Bührer C et al., Gestationsdiabetes mellitus (GDM) - Diagnostik, Therapie u. Nachsorge - Praxisleitlinie der Deutschen Diabetes-Gesellschaft (DDG) und der Deutschen Gesellschaft für Gynäkologie und Geburtshilfe (DGGG), Journal:Diabetologie und Stoffwechsel, Herausgeber M. Kellerer, S. Matthaei, Thieme Verlag, 2014; Seite S202-S213

21 Kleinwechter H, Schäfer-Graf U, Bührer C et al., Evidenzbasierte Leitlinie zu Diagnostik, Therapie u. Nachsorge, der Deutschen Diabetes Gesellschaft (DDG) und der Deutschen Gesellschaft für Gynäkologie und Geburtshilfe (DGGG), Herausgeber M. Kellerer, S. Matthaei; 2011

22 Koletzko B, Brands B, Poston L, Godfrey K, Demmelmair H. Early nutrition programming of long-term health. Proc Nutr Soc. 2012; 71(3): 371-378

23 Koletzko B (a) The power of programming: developmental origins of health and disease. Am J Clin Nutr 2011; 94: 1747S-2043S

24 Koletzko B (b) Symonds ME, Olsen SF. Programming research: where are we and where do we go from here?. Am J Clin Nutr. 2011; 94 (6 Suppl): 2036S2043S

25 Lacroix et al. Maternal/Fetal determinants of Insulin resistance in women during pregnancy and in offspring over life. Curr Diab Rep. 2013; 13 (2): 238-244

26 Lawlor DA, Fraser A, Lindsay RS, Ness A, Dabelea D, Catalano P, Smith GD, Sattar N, Nelson SM. Association of existing diabetes, gestational diabetes and glycosuria in pregnancy with macrosomia and offspring body mass index, waist and fat mass in later childhood: findings from a prospective pregnancy cohort. Diabetologia 2010; 53: 89-97

27 Metzger BE, Gabbe SG, Persson B et al. International association of diabetes and pregnancy study groups recommendations on the diagnosis and classification of hyperglycemia in pregnancy. Diabetes Care 2010; 33: 676-682

28 Plagemann A (a). Perinatal programming and functional teratogenesis: impact on body weight regulation and obesity. Clinic of Obstetrics 2005; 15; 86 (5): 661-668 
29 Plagemann A. (b) Fetale Programmierung und funktionelle Teratologie, Molekularmedizinische Grundlagen von fetalen und neonatalen Erkrankungen (Ganten D, Ruckpaul W; Hrsg.), Springer, Berlin, 2005, 325-342

30 Plagemann A, Harder T, Rake A, Melchior K, Rittel F, Rohde W, Dorner G. Hypothalamic insulin and neuropeptide $\mathrm{Y}$ in the offspring of gestational diabetic mother rats. NeuroReport 1998, 4069-4073

31 Ruemmele FM. Early programming effects of nutrition - life-long consequences? Ann Nenceutr Metab 2011; 58 (Suppl 2): 5-6

32 Schwartz MW, Woods SC, Porte Jr D, Seeley RJ, Baskin DG. Central nervous system control of food intake. Nature 2000; 404: 661-671

33 Silverman BL, Metzger BE, Cho NH, Loeb CA. Impaired glucose tolerance in adolescent offspring of diabetic mothers. Diabetes Care 1996; 18: 611-617

34 WHO. Definition, diagnosis and classification of diabetes mellitus and its complications. Report of a WHO consultation. Part 1: diagnosis and and classifications of diabetes melltitus. Geneva: World Health Organization and Department of Noncommunicable Disease Surveillance,Geneva,1999. Internet: http://whqlibdoc.who.int/ hq/1999/who_ncd_ncs_99.2.pdf (Stand: 11.09.2013) 